\title{
Bifurcations, basin erosion and dynamic integrity in a single-mode model of noncontact atomic force microscopy
}

\author{
V. Settimi ${ }^{1}$ and G. Rega ${ }^{1}$ \\ ${ }^{1}$ Dipartimento di Ingegneria Strutturale e Geotecnica, Università di Roma “La Sapienza”, 00197 Rome, Italy
}

\begin{abstract}
The nonlinear dynamical behavior of a single-mode model of noncontact AFM is analyzed in terms of attractors robustness and basin integrity. The model considered for the analyses, proposed by Hornstein and Gottlieb [7], consistently includes the nonlinear atomic interaction and the scan control, which appears as parametric excitation. Local bifurcation analyses are carried out to define the overall stability boundary in the excitation parameter space as the envelope of system local escapes, to be compared with the one obtained via numerical simulations. The dynamical integrity of the periodic bounded solutions is studied, and basin erosion is evaluated by means of IF and GIM integrity measures. The obtained erosion profiles allow us to dwell on the possible lack of homogeneous safeness of the stability boundary in terms of attractors robustness and to identify practical escape thresholds ensuring an a priori design safety target.
\end{abstract}

\section{Introduction}

Since the Binnig invention in 1986, the atomic force microscope (AFM) has proven to be a powerful instrument for the study of surfaces at the nanoscale resolution. Up to now, several models have been proposed to describe the AFM cantilever motion and the relationship between cantilever response and tip-sample interactions, whose characterization is fundamental to obtain information about surface topography and material properties [1]. AFM methods include static (contact) modes and dynamic (tapping, noncontact) modes; the latter ones require the modeling of attractive and repulsive interaction regimes, which show nonlinear behavior and make the model description very complicated. For this reason, point-mass models have been widely used in the investigation of cantilever dynamics in tapping and noncontact modes, while only few models treat the continuous system with a consistent nonlinear interaction [2]. Moreover, among several numerical analyses accomplished to describe the AFM nonlinear dynamic behavior, a lack of applications concerning attractors and basins of attraction identification, and their evolution through local bifurcations and basin erosion profiles, can be pointed out. These analyses, which are also tools for the evaluation of system dynamical integrity [3,4], are subjects of theoretical and practical importance, as it's nowadays agreed that the safety of a nonlinear system depends not only on the stability of its solution but also on the uncorrupted basin surrounding each solution $[5,6]$.
The aim of this paper is to investigate the dynamical integrity of a nonlinear continuum AFM model, with the attempt to achieve a detailed description of system nonlinear dynamics in terms of safety with respect to escape. The reference model, proposed by Hornstein and Gottlieb [7], is a single mode model of noncontact AFM, which consistently incorporates the generalized forces describing motion control as periodic boundary conditions, and the tip-sample interaction as localized nonlinear atomic interaction force. The analyses are focused on the influence of the parametric scan excitation on the dynamic behavior of the system.

A brief description of the model mathematical formulation is presented in Section 2, and its strongly nonlinear dynamics is analyzed in Section 3. Numerical analyses are carried out to detect the local bifurcation scenario which characterizes the model nonlinear dynamics in a wide range of forcing frequencies, with the scan excitation being chosen as varying parameter. The envelope of the obtained local escape thresholds determines the stability boundary in the excitation parameter space corresponding to safe basin annihilation. Evolution of periodic solutions basins of attraction is then studied as a function of increasing forcing amplitude, and integrity measures $[4,5]$ are computed to quantify their basin erosion levels and to identify the erosion profiles. Integrity evaluation is finally used to detect different frequency-dependent thresholds by assigning an a priori safety target which allows to select the level of system robustness to be considered acceptable for the desired model performances. 


\section{Dynamic problem formulation}

Consider a planar, inextensible and horizontal microbeam of length $L$ with a sharp tip close to its free end of height $h_{T}$, and with a distance $g$ between its fixed side and the sample. The beam material is considered to be linearly elastic, homogeneous and isotropic with Young's modulus $E$. Following the formulation of Hornstein and Gottlieb [7] and employing the extended Hamilton principle [8], the set of two coupled partial differential equations for the horizontal motion and the vertical transverse vibration is

$$
\begin{aligned}
& m \bar{u}_{t t}-\left[E I \bar{v}_{r r r} \bar{v}_{r}-J_{z} \bar{v}_{t t r} \bar{v}_{r}+\lambda\left(1+\bar{u}_{r}\right)\right]_{r}=\bar{Q}_{u} \\
& m \bar{v}_{t t}-\left[-E I\left(\bar{v}_{r r r}+\bar{v}_{r} \bar{v}_{r r}^{2}\right)+J_{z}\left(\bar{v}_{t t r}+\bar{v}_{t r}^{2} \bar{v}_{r}\right)+\lambda \bar{v}_{r}\right]_{r}=\bar{Q}_{v}
\end{aligned}
$$

where $\bar{u}(r, t)$ and $\bar{v}(r, t)$ are horizontal and vertical displacements and subscript letters denote partial differentiation with respect to arc length $r$ and time $t$. Coefficients $E I, J_{z}$ and $m$ are beam stiffness, principal moment of inertia and mass per unit length respectively, and $\lambda$ is a Lagrangian multiplier describing the fixed length of the beam.

Generalized forces in horizontal and vertical direction are represented by $\bar{Q}_{u}, \bar{Q}_{v}$, and are composed as follows

$$
\begin{aligned}
& \bar{Q}_{u}=\bar{g}_{1} \bar{u}_{t}-\bar{g}_{2} \bar{u}_{t r r}-\bar{g}_{3} \bar{u} \\
& \bar{Q}_{v}=\delta\left(r-a_{T}\right) F_{v}^{A}-d \bar{v}_{t}
\end{aligned}
$$

where $\bar{g}_{1}, \bar{g}_{2}$ and $\bar{g}_{3}$ are three terms related to linear viscous damping, material viscoelastic damping and a displacement gain respectively, while $d$ is a damping coefficient and $F_{v}^{A}$ is the atomic force derived from a Lennard Jones potential for a sphere-plane system [9], localized at the tip and representing the interaction between the tip and the sample.

A set of boundary conditions completes the formulation of the problem

$$
\begin{array}{lll}
\bar{v}(0, t)=0, & \bar{v}_{r r}(L, t)=0, & \bar{u}(0, t)=\bar{U}(t), \\
\bar{v}_{r}(0, t)=0, & \bar{v}_{r r r}(L, t)=0, & \bar{u}_{r}(L, t)=0
\end{array}
$$

with $\bar{U}(t)$ the horizontal scan displacement.

\subsection{Single mode model}

Following Hornstein and Gottlieb [7], the incorporation of the holonomic constraint, the isolation and expansion of the Lagrange multiplier and the nondimensionalisation and use of a moving reference frame lead to the formulation of an initial-boundary value problem with a homogeneous set of boundary conditions for the transverse vibrating motion. A single mode assumption and a Galerkin approximation (the basis function being that of a clamped-spring beam) reduce the IBVP to a second order differential equation, which has the following nondimensional form

$$
\begin{aligned}
& \ddot{x}\left(1+\alpha_{2} x^{2}\right)+\alpha_{1} x+\alpha_{2} x \dot{x}^{2}+\alpha_{3} x^{3}= \\
& -\Gamma_{1}(1+x)^{-2}-\rho_{1} \dot{x}-\rho_{2} \dot{x} x^{2} \\
& +\left(x \mu_{1}+\mu_{2} x^{3}\right)\left(\ddot{U}_{g}+\eta_{1} \dot{U}_{g}+\eta_{2} U_{g}\right)
\end{aligned}
$$

Here, $\Gamma_{1}$ is the atomic force interaction parameter, $\rho_{1}$ is the damping and $U_{g}$ is the horizontal excitation that is supposed to be harmonic $\left(U_{g}=U \sin \left(\omega_{u} t\right)\right) ; \eta_{1}$, $\eta_{2}$ and $\rho_{2}$ are feedback control parameters. For the sake of simplicity, feedback control and the nonlinear term related to $\alpha_{2}$ are neglected, to obtain

$$
\ddot{x}+\alpha_{1} x+\alpha_{3} x^{3}=\frac{-\Gamma_{1}}{(1+x)^{2}}-\rho_{1} \dot{x}-x \mu_{1} U \omega_{u}{ }^{2} \sin \left(\omega_{u} t\right)
$$

which describes the dynamics of a nonlinear microcantilever with a localized atomic interaction subject to a parametric excitation.

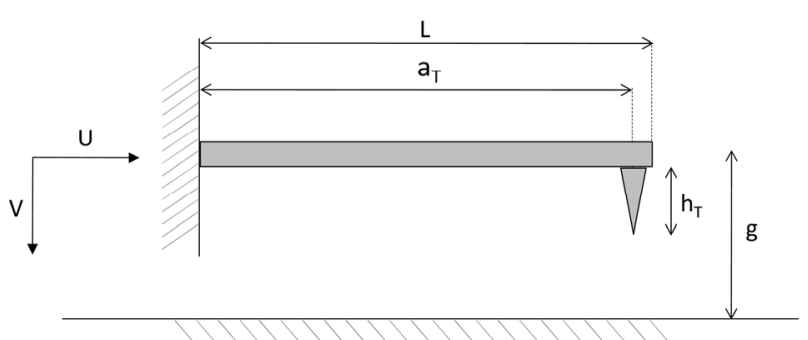

Fig.1. Microbeam parameters

\section{Nonlinear dynamic analysis}

\subsection{Bifurcation diagrams and stability boundaries}

The strongly nonlinear dynamics of the one-mode model has been analyzed making use of continuation techniques and numerical methods, for the following set of parameter values:

$\alpha_{1}=1, \alpha_{3}=0.1, \rho_{1}=0.001, \Gamma_{1}=0.1, \mu_{1}=1.5708$.

The calculated natural frequency for these values is $\omega_{1}=0.835$.

To determine the system stability boundary, several bifurcation diagrams at different forcing frequencies have been obtained and the main local bifurcations and periodic solutions have been detected. These maps are obtained by increasing slowly the forcing amplitude while holding the frequency constant. The results are summarized in figure 2. The system escape threshold (black curve) in the excitation parameter control space (forcing frequency vs forcing amplitude) separates the bounded solutions (below the curve) from the unbounded ones (above the curve) and is obtained as the envelope of local bifurcation escape thresholds in different parameter 

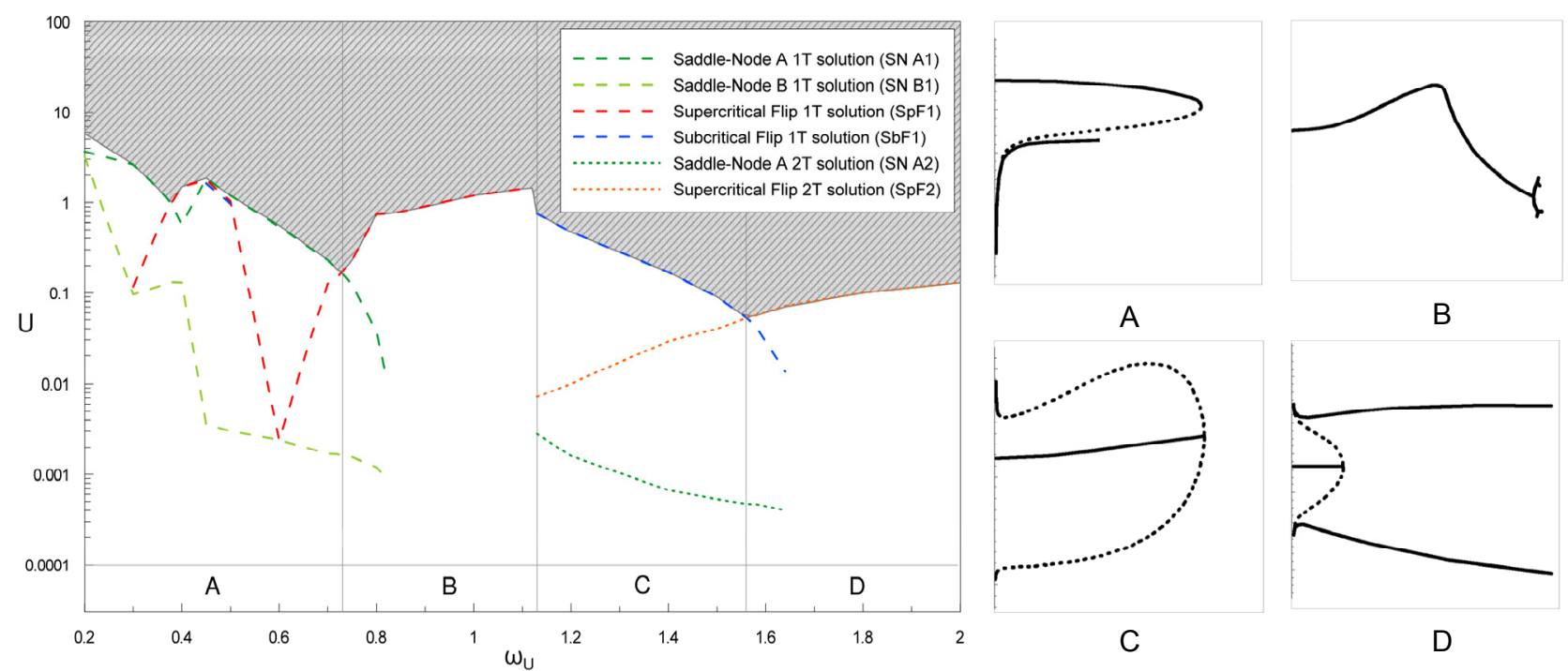

Fig. 2. Local bifurcations map for the frequency range $\omega_{u}=0.2-2$ and bifurcation diagrams showing the four different escape events in the frequency range analyzed: saddle-node escape (A), supercritical flip escape (B), subcritical flip (C) and supercritical flip of a 2 period solution (D)

ranges. The escape excitation amplitude corresponds to total annihilation of all basins of attraction and, from a physical viewpoint, represents the amplitude value that would bring the beam tip oscillation beyond the location of the sample (at $x_{e}=-1$ ).

It's worth noting that changes in the stability boundary slope correspond to changes in the kind of bifurcation event leading to escape and, apart from a localized sudden change in the frequencies range between 0.4 and 0.5 , four main different regions in the parameter control space can be picked out. At low frequencies, a saddle node bifurcation leads to disappearance of the sole existing 1-period solution. In the primary resonance region (around $\omega_{1}=0.835$ ) a sequence of period doubling events occurs in a narrow range of amplitudes and a supercritical flip bifurcation of the 1-period solution determines the disappearance of all periodic solutions. As the frequency increases, a subcritical flip bifurcation arises and the disappearance of a stable 1period solution together with the onset of two unstable 2period solutions brings to escape. At higher frequencies the escape bifurcation event is represented by a supercritical flip of a 2-period solution.

Figure 3 shows an example of bifurcation diagram at $\omega_{u}=0.8$, obtained by continuation techniques and using Floquet theory for the identification of the local bifurcation type. Around the primary resonance frequency, the system initially displays a stable lowamplitude 1-period P1 solution, which becomes unstable at $U=0.037$ and is connected to the high-amplitude 1period P1'solution through a saddle-node bifurcation. The P1' solution exists while the forcing amplitude increases up to the escape value $U=0.74$, where a supercritical flip bifurcation (SPF1 point) marks out the annihilation of all bounded solutions. In a narrow region between $\omega_{u}=0.5$ and $\omega_{u}=0.6$ a 6-period P6 solution arises and disappears through a supercritical flip bifurcation (SPF6 point).

Basins of attraction of $\mathrm{P} 1$ and $\mathrm{P} 1$ ' solutions at the forcing amplitude value $U=0.01$ are shown in figure 4a. The white area corresponds to unbounded solutions while red and blue basins confirm, as already suggested by the bifurcation map, that a competing P1' solution is growing up inside the dying P1 basin. The Poincaré map with trajectories in the phase plane and the temporal evolution of the two solutions are reported in figures $4 b, c$ and provide information about their different amplitude.

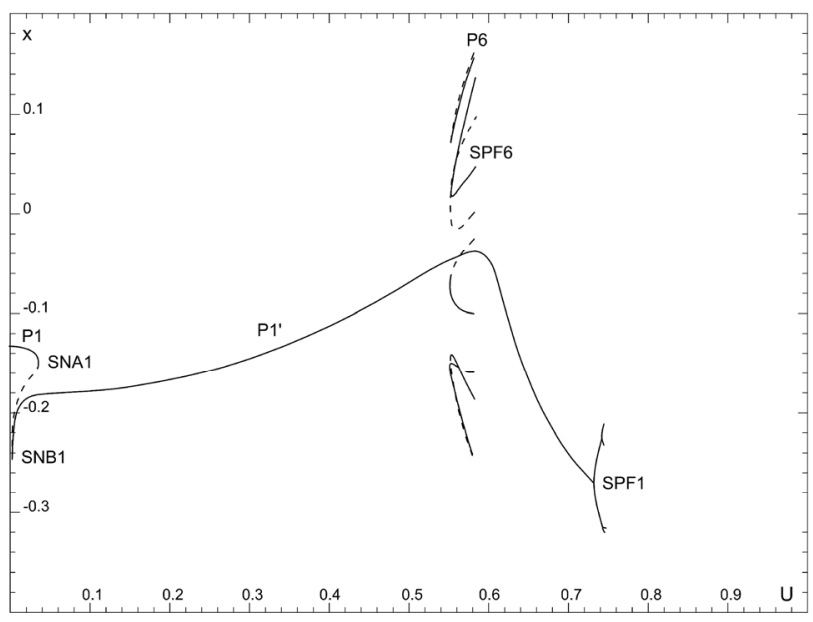

Fig. 3. Bifurcation diagram at $\omega_{u}=0.8$ 


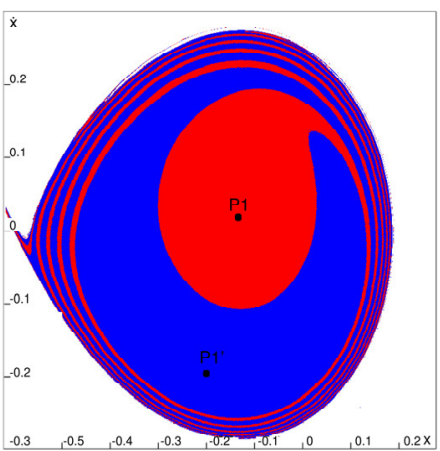

(a) (b)

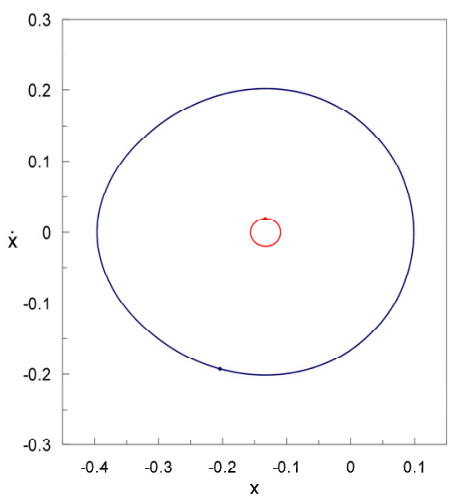

(c)

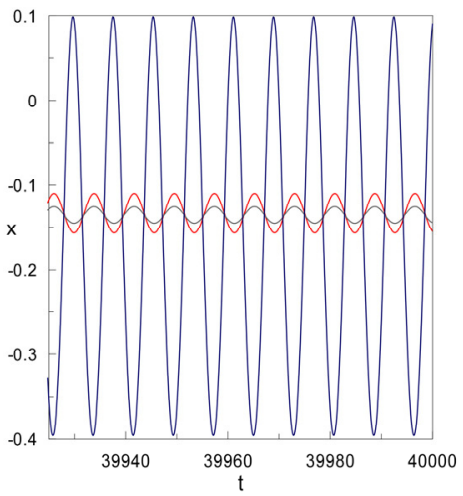

Fig. 4. Basins of attraction (a), Poincaré maps (b) and temporal evolutions (c) of P1 and P1' solutions at $U=0.01$

\subsection{Dynamic integrity and basin erosion}

As already mentioned, the escape profile obtained by mapping several bifurcation diagrams coincides with the forcing amplitude values at which basin annihilation occurs. Such stability boundary doesn't furnish any information about the erosion process of solution basins, which constitutes a critical issue corresponding to impending system failure, and therefore it has no practical utility from a safety viewpoint.

A fundamental tool for a complete description of the evolution of system dynamics up to escape is represented by the dynamical integrity concept, which allows us to determine and quantify the basins erosion level in view of detecting a practical safety threshold for the system [5]. The investigation of basin evolution due to system parameter variation and the subsequent construction of the so-called erosion profiles [4,5] are carried out by means of specific computational tools, based on the safe basin and integrity measure concepts. In this work, the safe basin is defined as the union of all basins of attraction of bounded solutions, with the transient dynamics being ignored, while the integrity indicators used to detect the erosion profiles are the global integrity measure (GIM), which represents the normalized hypervolume (area in 2D) of the safe basin, and the integrity factor (IF), which is the normalized radius of the largest hyper-sphere (circle in 2D) entirely belonging to the safe basin [4]. The phase space window is $x \in[-0.3,0.3]$ and $\dot{x} \in[-0.65,0.25]$, and contains the compact part of the basin of each attractor involved in the analysis.

Figure 5 shows the basins evolution at $\omega_{u}=0.8$ of all the periodic growing and dying solutions, with the representation of the IF circle relevant to the safe basin. At $U=0.005$ the safe basin starts to be corrupted by the unbounded solution basin of attraction (white area) and the erosion carries on as $U$ increases up to the basins annihilation at $U=0.75$. A 6-period solution arises and dyes around $U=0.6$. As already seen in the bifurcation diagram, two competing stable solutions are present at low amplitude values, with the original P1 solution (red basin) being eroded by the growing P1' one (blue basin) up to the P1 basin disappearance at $U=0.04$. The integrity analysis for this range of amplitude values is reported in figure 6a, where GIM and IF have been calculated and the erosion profiles of P1 and P1' solutions are plotted (green and blue lines, respectively) together with those of the safe basin (red lines). The integrity curves highlight a different behavior of the two integrity measures depending on whether the analysis refers to the competing $\mathrm{P} 1$ and $\mathrm{P} 1$ ' attractors or to the safe basin. In the first case, in fact, IF appears to be more sensitive to basin size variations than GIM, while in presence of the "infinity" attractor surrounding the safe basin GIM turns out to be more conservative than IF. This quantitatively different result is due to a qualitatively different kind of erosion process the basins are involved with: in the case of two different basins in the same well, the rolling up arise of P1' solution causes an instantaneous reduction of the circle measuring the magnitude of the compact part of the P1 basin (IF), while affecting the basin volume decrease (GIM) in a minor way. On the contrary, the safe basin erosion is due to the penetration of fractal tongues which are localized in the basin boundaries and do not strongly modify the basin compact core.
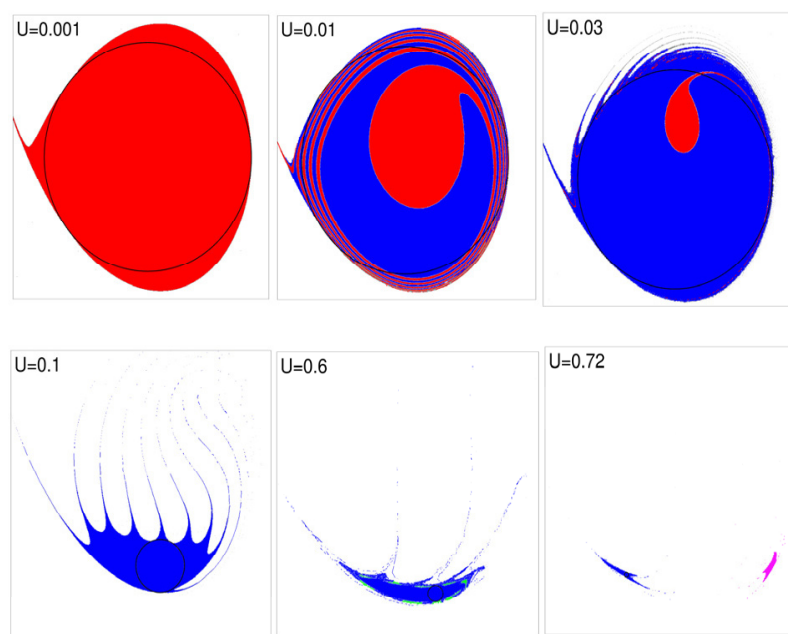

$\mathrm{U}=0.72$

Fig. 5. Basins evolutions at $\omega_{u}=0.8$. Black circles are the safe basin IF measure. 
(a)

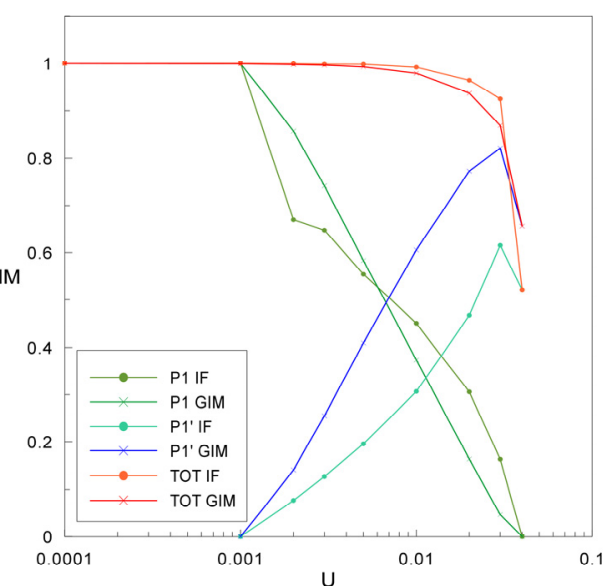

(b)

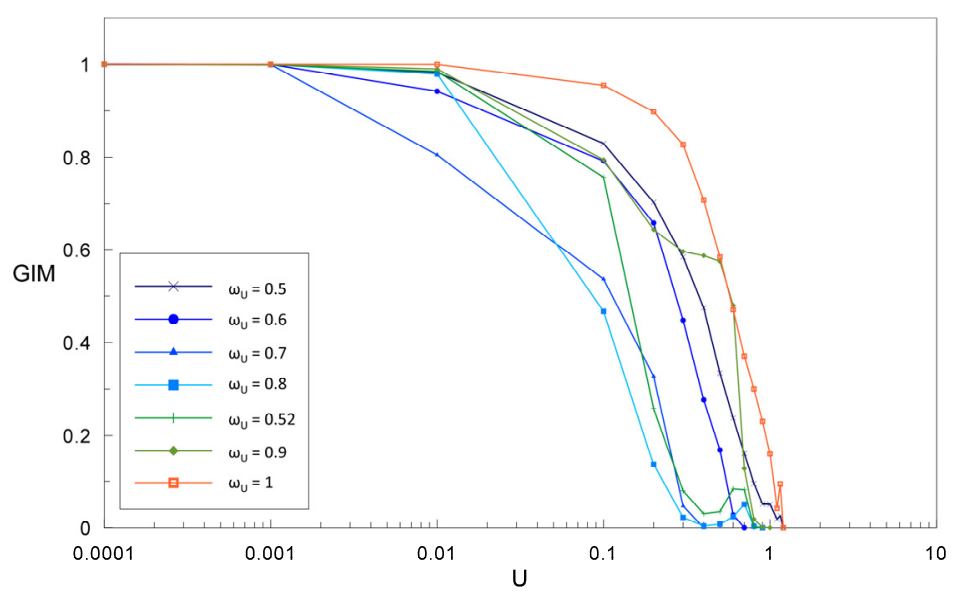

Fig. 6. Erosion profiles of periodic solutions basin and of safe basin for a fixed frequency $\left(\omega_{u}=0.8\right)$ (a) and of safe basin for several forcing frequencies (b)

Figure $6 \mathrm{~b}$ represents a summary diagram of GIM profiles of the safe basin for different forcing frequencies in the range $0.5-1$. The profiles have the classical qualitative behavior of the so-called "Dover cliff" erosion curve [4], which is characterized by a slow decrease of the un-eroded volume of the safe compact region, followed by a suddenly fall down to zero. It is worth noting that at $U=0.01$ the erosion profiles show meaningfully different slopes, namely in the forcing frequency range $0.5-0.7$ they undergo a remarkable decrease, while for higher frequencies the decrease is definitely lower. This behavior can be explained by analyzing the basin evolution at the selected excitation amplitude: a new external, i.e. disconnected, competing attractor arises at $\omega_{u}=0.55$ and gradually reduces the existent basin as the frequency increases, up to $\omega_{u}=0.73$ where the interpenetration of the two basins in the same well occurs thus enlarging the safe basin.

\subsection{Theoretical vs practical stability boundaries}

A matter of theoretical and practical interest consists of the comparison between the stability boundary carried out by mapping the bifurcation diagrams $(b d)$, as previously presented, and the escape threshold obtained by numerical integration (ni) with fixed initial conditions [7]. The results are reported in figure $7 \mathrm{a}$, where the blue curve, related to the numerical simulation, represents the forcing amplitude values $U$, for several forcing frequencies, at which the divergence of system response occurs. In this case, the equilibrium of the Hamiltonian system has been selected as fixed initial conditions. The figure highlights a significant difference with respect to the $b d$ method (green curve), pointing out a few remarks. The $n i$ curve lies below the $b d$ curve in the whole frequency range, with a gap up to 250 times the $n i$ absolute value. Moreover, ni local minima occur at primary and secondary resonance frequencies, according to the 1-period solution chosen as initial conditions, while $b d$ minima are shifted due to the coexistence of solutions of different period. It's worth remarking that the $n i$ escape value strongly depends on the particular selection of the initial condition pair. Looking for instance at $\omega_{u}=0.8$, a slight increase of the excitation amplitude (around the escape value $U=0.03$ ) causes erosion of the basin just in the neighborhood of the initial condition (white point in figures $7 \mathrm{~b}, \mathrm{c}$ ), while leaving more than $50 \%$ of residual integrity of the safe basin. In contrast, actual basin annihilation ( $b d$ curve) occurs only at $U=0.74$. This clearly highlights how, in terms of general system safety with respect to escape, consideration of the outcome of a single trajectory may furnish misleading information, unless being specifically interested to the response ensuing from given initial conditions.

Even more important is that, in practical application, the integrity evaluation can be used to determine different frequency-dependent thresholds by assigning an a priori design safety target. Figure 8 shows four iso-integrity curves corresponding to increasing target values. Selecting for instance the $30 \%$ residual safety target, the corresponding red curve allows the critical discussion of the results obtained via numerical simulation (black curve), for frequencies ranging between 0.5 and 1. The relevant outcomes highlight a residual integrity strongly variable in the control parameter range, yielding in particular an over conservative escape threshold for the highest frequencies and a dangerous underestimate of the basin erosion effect for the lowest frequency values. 


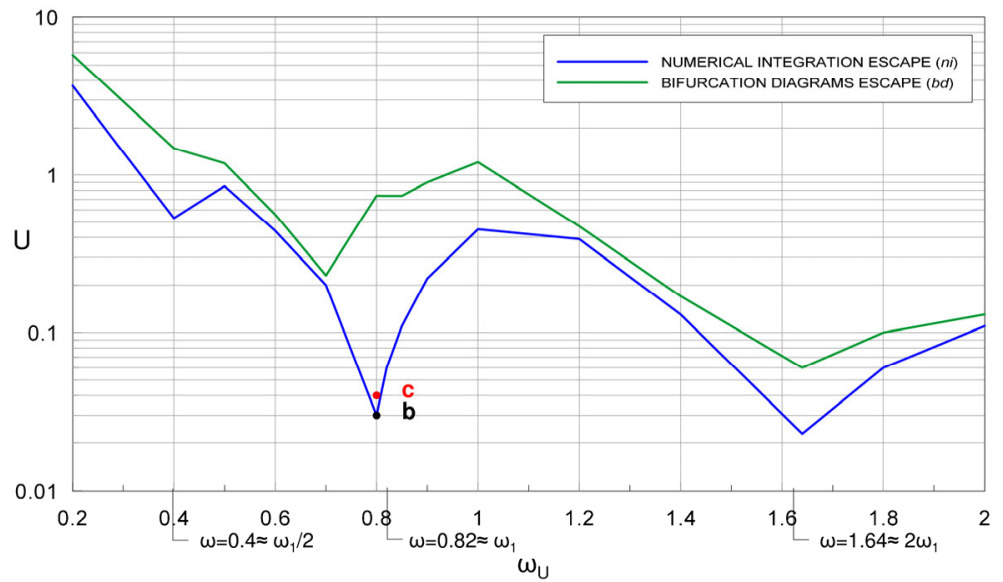

(b)

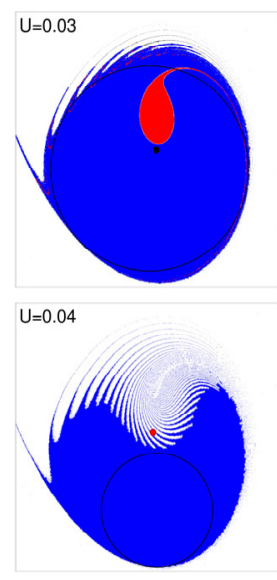

Fig. 7. Comparison between $n i$ (blue) and $b d$ (green) escape thresholds (a); state-space basin evolution at $\omega_{u}=0.8$ for $U=0.03$ (black point b) (b) and $U=0.04$ (red point c) (c)

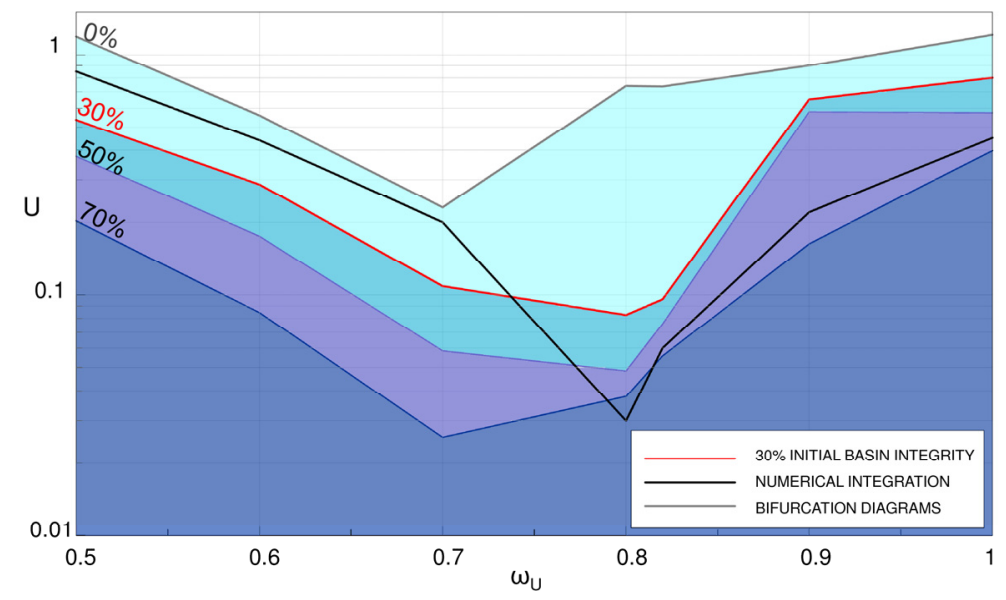

Fig. 8. Comparison between theoretical and practical boundaries

\section{Conclusions}

Local and global dynamics of a single mode model of noncontact AFM have been investigated in view of evaluating the system dynamical integrity. Extended numerical analysis has allowed to construct several bifurcation diagrams and to determine the system escape threshold in the excitation parameter space as the envelope of local bifurcation escape boundaries in various parameter ranges. The obtained escape excitation amplitudes, corresponding to basins total annihilation, have been compared with those obtained via numerical integration of the system equations with fixed initial conditions, which correspond to solution divergence. Thanks to the analysis of basin of attraction evolution and to the evaluation of different integrity measures (GIM and IF), erosion profiles have been obtained as a function of the increasing forcing excitation amplitude; this kind of analysis furnishes residual integrity levels associated with the system global dynamics. The results highlight a marked variability with respect to the stability boundary obtained via numerical integration and, mostly, a meaningful lack of homogeneous safeness of the latter, as regards robustness of the periodic solutions, in the excitation parameter space. In contrast, the analysis of basins of attraction evolution in state space and the construction of erosion profiles allow us to detect thresholds of residual integrity able to ensure acceptable safety targets established a priori according to the required system performances.

\section{References}

1. F.J. Giessibl, Reviews of modern physics, 75(3), 949-983 (2003)

2. Y. Song, B. Bhushan, Journal of Physics: Condensed Matter 20, 225012 (2008)

3. M.S. Soliman, J.M.T. Thompson, Journal of Sound and Vibration 135, 453-475 (1989)

4. G. Rega, S. Lenci, Nonlinear Analysis 63, 902-914 (2005)

5. G. Rega, S. Lenci, Journal of Vibration and Control 14, 159-179 (2008)

6. S. Lenci, G. Rega, International. Journal of Non-Linear Mechanics 46, 1240-1251 (2011)

7. S. Hornstein, O. Gottlieb, Nonlinear Dynamics 54, 93-122 (2008)

8. M.R.M. Crespo da Silva, C.C. Glynn, Mechanics Based Design of Structures and Machines 6(4), 437-448 (1978)

9. J. Israelachvili, Intermolecular and Surface Forces (Academic Press, London 1992) 